\title{
Effects of horse-riding therapy and rhythm and music-based therapy on functional mobility in late phase after stroke
}

\author{
Lina Bunketorp-Käll ${ }^{\mathrm{a}, \mathrm{b}, *}$, Marcela Pekna ${ }^{\mathrm{a}, \mathrm{c}}$, Milos Pekny ${ }^{\mathrm{a}, \mathrm{c}, \mathrm{d}}$, Christian Blomstrand ${ }^{\mathrm{a}, \mathrm{e}}$ \\ and Michael Nilsson ${ }^{\mathrm{a}, \mathrm{c}, \mathrm{f}, \mathrm{g}}$ \\ ${ }^{a}$ Center for Brain Repair, Department of Clinical Neuroscience, Institute of Neuroscience \\ and Physiology, Sahlgrenska Academy at the University of Gothenburg, Gothenburg, Sweden \\ ${ }^{\mathrm{b}}$ Center for Advanced Reconstruction of Extremities C.A.R.E. Institute of Clinical Sciences, \\ Sahlgrenska University Hospital/Mölndal, Sweden \\ ${ }^{\mathrm{c}}$ Florey Institute of Neuroscience and Mental Health, Parkville, Melbourne, Australia \\ ${ }^{\mathrm{d}}$ University of Newcastle, New South Wales, Australia \\ ${ }^{\mathrm{e}}$ Department of Clinical Neuroscience, Stroke Center West, Institute of Neuroscience \\ and Physiology, The Sahlgrenska Academy at the University of Gothenburg, Gothenburg, Sweden \\ ${ }^{\mathrm{f}}$ Centre for Rehab Innovations (CRI), University of Newcastle and Hunter Medical Research \\ Institute (HMRI) Newcastle, Australia \\ ${ }^{\mathrm{g}}$ LKC School of Medicine, Nanyang Technological University, Singapore
}

\begin{abstract}
.
BACKGROUND: Persons with stroke commonly have residual neurological deficits that seriously hamper mobility.

OBJECTIVE: To investigate whether horse-riding therapy (H-RT) and rhythm and music-based therapy (R-MT) affect functional mobility in late phase after stroke.

METHODS: This study is part of a randomized controlled trial in which H-RT and R-MT was provided twice weekly for 12 weeks. Assessment included the timed 10-meter walk test (10 mWT), the six-minute walk test (6 MWT) and Modified Motor Assessment Scale (M-MAS).

RESULTS: 123 participants were assigned to H-RT $(n=41)$, R-MT $(n=41)$, or control $(n=41)$. Post-intervention, the H-RT group completed the $10 \mathrm{mWT}$ faster at both self-selected $(-2.22$ seconds [ $95 \% \mathrm{CI},-3.55$ to -0.88$] ; p=0.001)$ and fast speed $(-1.19$ seconds $[95 \% \mathrm{CI},-2.18$ to -0.18$] ; p=0.003)$, with fewer steps $(-2.17$ [95\% CI, -3.30 to -1.04$] ; p=0.002$ and -1.40 [95\% CI, -2.36 to -0.44$] ; \mathrm{p}=0.020$, respectively), as compared to controls. The H-RT group also showed improvements in functional task performance as measured by M-MAS UAS (1.13 [95\% CI, 0.74 to 1.52]; $\mathrm{p}=0.001)$. The gains were partly maintained at 6 months among H-RT participants. The R-MT did not produce any immediate gains. However, 6 months post-intervention, the R-MT group performed better with respect to time; -0.75 seconds $[95 \%$ CI, -1.36 to -0.14$] ; p=0.035$ ) and number of steps -0.76 [95\% CI, -1.46 to -0.05$] ; \mathrm{p}=0.015$ ) in the $10 \mathrm{mWT}$ at self-selected speed.

CONCLUSIONS: The present study supports the efficacy of H-RT in producing immediate gains in gait and functional task performance in the late phase after stroke, whereas the effectiveness of R-MT is less clear.
\end{abstract}

Keywords: Stroke, rehabilitation, randomized controlled trial, horse-riding therapy, music therapy

\footnotetext{
*Address for correspondence: Lina Bunketorp Käll, Ph.D., Assoc. Prof, Centre for Advanced Reconstruction of Extremities, Sahlgrenska University Hospital/Mölndal, House U1, 6th floor,
}

43180 Mölndal, Sweden. Tel.: +46 709 723101; Fax: +46 31 873009; E-mail: lina.bunketorp-kall@neuro.gu.se. 


\section{Introduction}

Advances in diagnosis and acute treatment lead to increasing number of stroke survivors and consequently increase the demand on rehabilitation centres, families, and other caregivers (Adamson et al., 2004; Corbyn, 2014). The neurological deficits resulting from stroke can seriously hamper postural control functions and gait (Daly \& Ruff, 2007). Such mobility impairments commonly also affect patients' ability to maintain social engagement (Alzahrani et al., 2011; Michael, 2002; Rand et al., 2009). Sedentary lifestyle and social isolation are associated with numerous detrimental health conditions that impact on well-being, and quality of life of stroke survivors. Stroke rehabilitation therefore faces the challenge of finding therapeutic approaches that optimize motor recovery after stroke (Bernhardt et al., 2017b; Bernhardt et al., 2017c), as reflected by the growing number of studies evaluating promising interventions (Veerbeek et al., 2014).

Functional mobility is increasingly used as an outcome in clinical studies as it may provide a functional measure of the overall health condition (e Silva et al., 2017; Tyson \& Rogerson, 2009). Functional mobility involves person's ability to move independently in a variety of environments in order to participate in the activities of daily living or to perform functional activities such as bed mobility, transfers, ambulation or specific tasks (Choi et al., 2015). Enhanced gait capacity is one of the most important goals of stroke survivors (Harris \& Eng, 2004), and gain in gait speed resulting from the application of therapeutic approaches is a variable of major interest in clinical studies (Dickstein, 2008). Improving mobility is of great importance for achieving independence and the ability to perform daily activities, as well as for re-integration in the community (Jette et al., 2005). It is suggested that improvement in walking speed reflects a genuine improvement in mobility, even if other mostly categorical measures fail to detect it (Kollen et al., 2006). Repeated assessment with a simple timed walking test has been validated as a responsive method to detect change in walking performance over time (Collen et al., 1990). Additional gait parameters such as step length, and step rate or cadence have often been analysed in such studies, including stroke survivors (Hesse et al., 2001; Kollen et al., 2006; Öberg et al., 1993). Significant correlations have been found between walking speed, step length and step frequency in individuals after stroke
(Brandstater et al., 1983; Hesse et al., 2001; Wall \& Turnbull, 1986).

As a growing number of studies challenge the concept of reaching a plateau in stroke recovery (Demain et al., 2006), there is a need to design and evaluate interventions that support continuous stroke recovery (Bernhardt et al., 2017b; Bernhardt et al., 2017c). Collaborative multidisciplinary research programs that span the complex stroke continuum need to be developed with the ultimate goal of reducing the burden of stroke (Bernhardt et al., 2017a). Growing evidence points to the combination of therapeutic modalities as the key to maximal functional recovery after stroke (Corbett et al., 2015; Mala \& Rasmussen, 2017). This treatment strategy is also referred to as multimodal therapy.

We previously reported that emotionally engaging multimodal interventions incorporating horse-riding therapy (H-RT) and rhythm and music-based therapy (R-MT) have potential to influence perceived recovery following stroke (Bunketorp-Käll et al., 2017). Both therapeutic modalities incorporate motor, sensory and cognitive stimulation with the goal of promoting recovery after stroke. Both interventions contributed to gains in balance, and H-RT also led to improved functional balance, as measured by the Timed Up and Go test. To enable analyses with respect to the impact of the interventions on gait and functional task performance, additional secondary measures were included in the trial (Bunketorp-Käll et al., 2017; Bunketorp Kall et al., 2012). In the present study, we report on the effects of these interventions delivered in the late phase of stroke on participants' gait capacity and functional task performance. Given the explicit motor and sensory inputs of therapeutic riding that are thought to facilitate gait in neurologically disabled individuals, we hypothesised that the H-RT would significantly improve gait capacity.

\section{Methods}

\subsection{Study design and participants}

The present study is a part of a three-armed randomized controlled trial, the study protocol, and recruitment procedure of which were previously described (Bunketorp Kall et al., 2012). The eligibility criteria were limited to individuals who suffered from stroke 10 months to 5 years prior to their poten- 
tial inclusion in the trial. Selection criteria for trial eligibility are presented in Table 1. Participants were consecutively and randomly allocated to one of three parallel groups: H-RT, R-MT or a control group. Participants in the control group received the R-MT intervention a year later. The study was conducted according to the Consolidated Standards of Reporting randomized Trials (CONSORT) (Schulz et al., 2010). Evaluation was conducted prior to and directly after the 12-week long intervention, as well as three and six months post-intervention. All participants signed

Table 1

Selection criteria for trial eligibility

- Aged 50-75 years

- Disability grade 2 or 3 on $\mathrm{mRS}^{*}$

- Chronic impairment after stroke (minimum 10 months to maximum 5 years)

- Ischaemic or haemorrhagic stroke or subarachnoid haemorrhage with initial presence of hemispheric impact/symptoms

- Ability to understand written and oral information and instructions in Swedish

- Live in their own home

- Able to travel to the place of intervention and evaluation

- No need for personal assistance in the following activities of daily living while participating in the treatment (going to the toilet, transport/transportation services for disabled, walking)

- No pronounced fear of horses or allergy constituting a risk for the patients to participate in the therapeutic riding

- No heart conditions constituting a risk for the individual to participate in the interventions

- No history of non-controlled epileptic seizures constituting a risk for the patients to participate in the interventions

- No lack of cognitive and/or verbal ability or visual impairment that would make it difficult for the individual to understand instructions and/or evaluation

- No total arm paralysis

- No injury, disease or addiction that would render the individual unsuitable for the trial

- Bodyweight $\leq 97 \mathrm{~kg}$ (to optimise safe horseback riding)

- No more than half-time employment

- No participation in RMT or HRT $<10$ months prior to inclusion

- No additional stroke within the past year (TIA is however accepted)

- Accepting allocation to either of the three groups which might mean accepting staying without any of the treatment procedures for one year

*Modified Rankin Scale: An ordinal disability rating scale ranging from zero to $6(0=$ no symptoms $)$. mRS grade 1 : No significant disability despite symptoms; able to carry out all usual duties and activities; mRS grade $2=$ Slight disability: unable to carry out all previous activities but able to look after own affairs without assistance; $\mathrm{mRS}$ grade $3=$ Moderate disability: requiring some help, but able to walk without assistance; $\mathrm{mRS}$ grade 4: Moderately severe disability: unable to walk without assistance and unable to attend to own bodily needs without assistance; mRS grade 5: Severe disability: bedridden, incontinent and requiring constant nursing care and attention; mRS grade 6: Dead. a written informed consent form and were informed that they could withdraw from the study at any time. Ethical approval was granted by the Regional Ethical Review Board in Gothenburg (Ref number: 698-09) and the study is conducted in accordance with relevant ethical guidelines.

\subsection{Randomisation and blinding}

As gender and brain laterality might influence outcomes in clinical studies,(Johansson, 2012) the randomisation was stratified with respect to gender and hemispheric location of the stroke. A statistician performed computer-generated randomisation using random permuted blocks for each of the $2 \times 2$ strata. Block size was known only by the statistician who performed the randomisation. Another independent person sequentially numbered opaque envelopes, each of which contained the name of the intervention group that had been determined by the randomisation. The participants were consecutively recruited to the randomisation list by the project leader. The envelopes were opened in sequential order as each participant entered the trial. Randomisation codes were not accessed until all measurements were completed. Assessors were blinded to treatment allocation. Due to the nature of the therapy programs, blinding of participants and treating therapists was not feasible. Data analysts were not blinded. To keep assessors blinded, participants and intervention therapists were instructed not to reveal treatment allocation or participants' study experiences to the assessors.

\subsection{Interventions}

During the 12 weeks of intervention, participants attended two weekly sessions of the R-MT or H-RT. Both interventions have the common feature that they provide an enriched environment designed to stimulate motor, cognitive, sensory, and social functions. They differ, however, in terms of dosage, execution, activities, and targeted outcomes. A detailed description of the interventions as well as the specification of how the therapeutic modalities are expected to affect targeted outcomes are presented in Supplementary file 1. Participants in the delayed R-MT group (controls) were told not to start any new therapies during the duration of the study, but were allowed to continue with their regular activities and usual care such as outpatient physiotherapy, occupational therapy, or speech therapy. 


\subsection{Statistical analyses}

According the sample size calculation described previously (Bunketorp Kall et al., 2012), at least 41 patients were required in each of the three groups for the results to satisfy the power criteria of $80 \%$. Analyses were done according to intention-to-treat (ITT). In addition, a per-protocol analysis was performed. The outcome is analyzed in terms of change from baseline to each measurement point. Baseline and demographic characteristics are summarized using descriptive statistics. QQ-plots and histograms as well as skewness and kurtosis were used to assess whether the outcome variables approximated a normal distribution. Analyses of covariance (ANCOVA) with gain in each outcome measure as a dependent variable, initial score of each outcome measure as a covariate, and intervention group as factor, were done using SPSS v.22.0 (IBM Corp., Armonk, NY, USA). The results were considered significant if $P$ was less than .05 .

\section{Measures}

\subsection{Assessment of gait capacity}

To measure gait speed the timed 10 meter walk test (10 mWT) was used (Duncan et al., 2007) The test is performed on a 10-meter distance marked by tape on the floor. In the first round the subject was asked to walk at a self-selected walking speed. In the second round, they were instructed to walk as fast as they could. Walking aids were allowed. Walking time was measured in seconds. The number of steps was calculated and conversed into step length. In order to determine whether potential gains in terms of gait reached the level established as a meaningful change (Perera et al., 2006), as well as the suggested cut-off point for community ambulation (van de Port et al., 2008), gait speed was also expressed in meters per second, by dividing distance (in metres) by time (in seconds). The $10 \mathrm{mWT}$ has been widely used for locomotion studies involving various patient groups (Nagano et al., 2015). The test is considered to have high reliability and low variability as an interrater and test-retest (Perry et al., 1995; Tyson \& Connell, 2009).

After the first intervention group had been enrolled and randomized, the six-minute walk test (6MWT) was added to the outcome measures. The 6MWT is a sub-maximal exercise test used to assess walk- ing endurance (Cooper, 1968; Swisher \& Goldfarb, 1998).

\subsection{Observer-assessed functional mobility performance}

As a means of assessing everyday motor function in patients with stroke the Modified Motor Assessment Scale according to Uppsala University Hospital (MMAS UAS) was used (Andersson \& Clevnert, 1997). The M-MAS UAS is a modification of the original MAS described by Carr et al. (Carr et al., 1985). The instrument is based on the Motor Assessment Scale (MAS) which is a performance-based scale based on a task-oriented approach that assesses performance of functional tasks rather than isolated patterns of movement (Carr et al., 1985). The maximum score of 55 indicates good motor function. It has been modified and developed twice since 1991, as well as tested for reliability and validity in different studies (Poole \& Whitney, 1988). In this study, the M-MAS UAS version 1999 was be used (Barkelius et al., 1997). The M-MAS UAS measures motor control in eight levels: A: horizontal displacement, B: back lying to sitting movement, C: sitting down, D: sitting to standing, E: walking, F: arm functionality, G: hand movements and $\mathrm{H}$ : fine motor skills. The total M-MAS score was used to reflect a more global perspective of functional mobility capacity (e Silva et al., 2017; Tyson \& Rogerson, 2009).

\section{Results}

All 123 individuals (mean age of 63 years, age range 49-74) who were enrolled in the RCT were included in these analyses. The average number of days elapsed from the stroke insult was 1056 days (range: 318-3624). Exclusion and dropout throughout the RCT was reported previously (Bunketorp-Käll et al., 2017). Mean attendance rate at the H-RT and R-MT was $83 \%$ and $88 \%$ respectively, equivalent to at least 20 treatment sessions (SD 5.0) for the H-RT group and at least 21 treatment sessions (SD 4.0) for the R-MT group. Baseline demographic and clinical characteristics of trial participants are presented in Table 2. Demographic data were well balanced, with no differences between groups. Clinical characteristics indicated a trend towards greater mobility impairment in the H-RT group with respect to the timed $10 \mathrm{mWT}$ (ANOVA; $p=0.08$ ). No differences were observed between participants who 
Table 2

Demographic characteristics of study participants

\begin{tabular}{lccc}
\hline & R-MT $(n=41)$ & H-RT $(n=41)$ & Control $(n=41)$ \\
\hline Variable & & & \\
Sex: Women/Men $(\%)$ & $18(43.9) / 23(56.1)$ & $17(41.5) / 24(58.5)$ & $19(46.3) / 22(53.7)$ \\
Age in years & $62.7(6.7)$ & $62.6(6.5)$ & $63.7(6.7)$ \\
Years of schooling & $14.2(4.1)$ & $12.5(4.2)$ & $13.5(4.3)$ \\
Time since stroke onset in days & $969.8(422.9)$ & $1101.9(576.1)$ & $1096.3(439.0)$ \\
Site of the stroke lesion & & & \\
$\quad$ Right $(\%) / L e f t(\%)$ & $20(48.8) / 21(51.2)$ & $20(48.8) / 21(51.2)$ & $18(43.9) / 22(53.7)$ \\
Stroke type & & & \\
$\quad$ Haemorrhage (\%)/Infarct (\%) & $9(22.0) / 32(78.0)$ & $14(34.1) / 27(65.9)$ & $13(31.7) / 28(68.3)$ \\
Modified Rankin Scale & & & \\
$\quad$ Grade 2 (\%)/Grade 3(\%) & $24(58.5) / 17(51.5)$ & $23(56.1) / 18(43.9)$ & $25(61.0) / 16(39.0)$ \\
NIHSS & $3.0(2.9)$ & $2.7(3.1)$ & $2.8(3.6)$ \\
10 mWT Time (sec) & & & \\
$\quad$ Self-selected speed & $10.03(3.02)$ & $15.05(14.90)$ & $13.37(8.55)$ \\
$\quad$ Fast speed & $7.26(2.93)$ & $9.71(7.71)$ & $9.84(7.10)$ \\
6 MWT (meters) & $437.69(107.03)$ & $361.29(138.02)$ & $374.89(166.77)$ \\
M-MAS-UAS & $51.39(5.92)$ & $49.63(6.83)$ & $50.80(5.80)$ \\
\hline
\end{tabular}

Data are number (\%) or mean (SD). H-RT = Horse-riding therapy; R-MT = Rhythm and music-based therapy; NIHSS = The National Institutes Health Stroke Scales, 10 mWT: 10-meter walk test; 6 MWT: 6-minute walk test, M-MAS UAS = Modified Motor Assessment Scale (Uppsala University Hospital).

completed the intervention and those who did not (dropouts) with regard to baseline demographic and clinical characteristics. One participant in the R-MT died during the intervention period and was excluded from the analyses.

Changes in gait and mobility parameters for the three study groups at post-test and at the 6 months follow-up are summarized in Table 3. Immediately after intervention, the mean time to complete the 10-meter walk in self-selected speed for the H-RT and R-MT participants was reduced by $2.22( \pm 4.24)$ and $0.53( \pm 1.41)$ seconds, respectively, as compared to an increase of $0.37( \pm 4.13)$ in the control group (Fig. 1). The corresponding improvement for fast speed was $1.19( \pm 3.14)$ seconds in the H-RT group, while there was no change in RM-T group, and the control group performed worse compared to baseline (Table 3). Analyses with ANCOVA revealed that the improvements in the timed 10-meter walk at both self-selected and fast walking speed were significant ( $p=0.006$ and $p=0.011$, respectively). Further pairwise comparisons showed that these differences were ascribed to the H-RT group ( $p=0.001$ and $p=0.003$, respectively).

After the intervention, the groups differed also with respect to the number of steps taken during self-selected and fast 10-meter walk $(p=0.002$ and $p=0.020$, respectively). Further analysis showed that the difference post-intervention was ascribed to the H-RT group ( $p<0.001$ and $p=0.005$, respectively). When data derived from the $10 \mathrm{mWT}$ (self-selected and fast speed) was converted into step length, we did not find any between-group differences at postintervention. The increase in distance covered in the 6 MWT did not differ between the groups ( $p=0.177$ ). Improvements were also found in the performance of functional tasks as measured by M-MAS UAS $(p=0.001)$. Further pairwise comparisons showed that these differences were ascribed to the H-RT group $(p=0.001)$.

At 6 months, there was a significant between-group difference with respect to self-selected gait speed and step length during the timed 10-meter walk $(p=0.009$ and 0.031). Further pairwise comparisons showed that the increase in self-selected gait speed and step length were ascribed to both the R-MT ( $p=0.035$ and $0.047)$, and H-RT group ( $p=0.031$ and 0.013$)$. The between-group difference at 6 months for the timed 10-meter walk at fast walking speed remained significant in favor of the H-RT group $(p=0.011)$. At the 6 month follow-up, the differences between groups with respect to the number of steps taken during self-selected and fast 10-meter walk were sustained ( $p=0.001$ and $p=0.042$, respectively). Further analysis ascertained that the difference in the number of steps taken at self-selected speed was ascribed to both the H-RT and R-MT group $(p<0.001$ and $p=0.015$, respectively), whereas for fast speed, the difference was in favor of only the H-RT group $(p=0.003)$. The immediate gains in functional mobility performance among H-RT participants as measured by M-MAS UAS, were not sustained at 6 months. The results of 
Table 3

Changes in functional mobility performance for the study groups post intervention and at the 6-month follow-up according to ITT

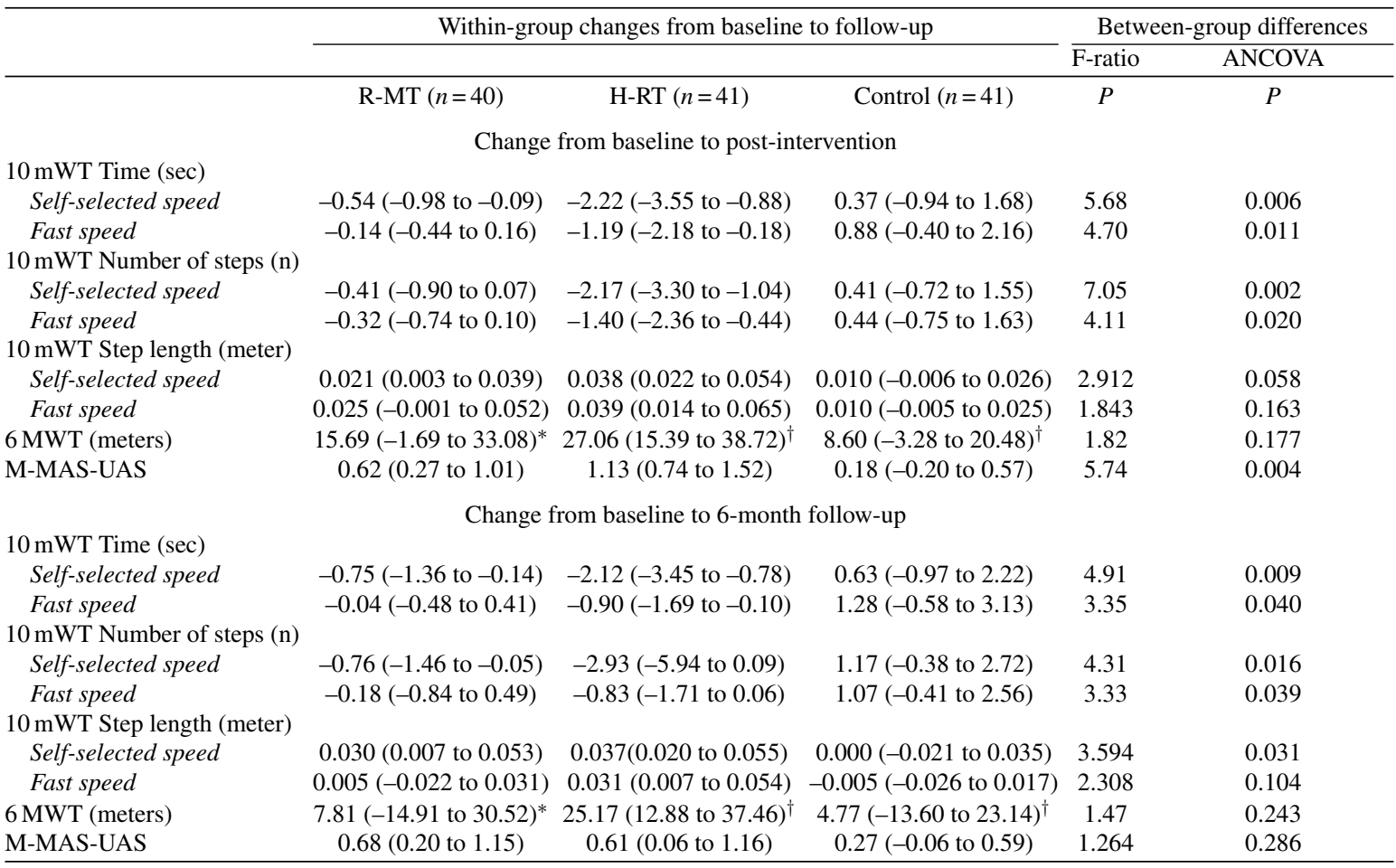

Data are mean $(95 \% \mathrm{CI})$. Data available for: ${ }^{*} 36$; ${ }^{\dagger} 35$; participants. $10 \mathrm{mWT}$ : 10 -meter walk test; $6 \mathrm{MWT}$ : 6-minute walk test, M-MAS UAS $=$ Modified Motor Assessment Scale (Uppsala University Hospital).

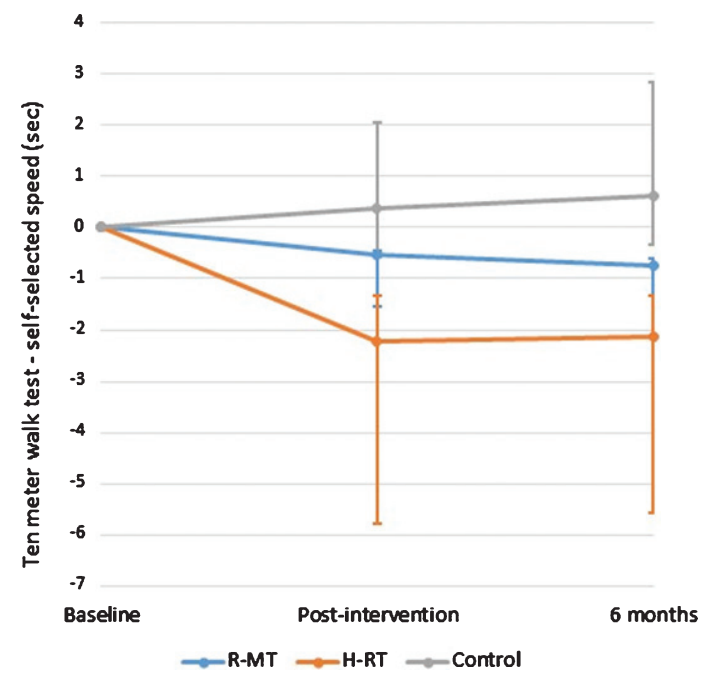

Fig. 1. The change in the 10-meter walk test in self-selected speed from baseline to each evaluation point presented as mean $(95 \%$ confidence interval).

the additional per-protocol analysis were consistent with those of the analysis according to ITT.

\section{Discussion}

This study shows that a heterogeneous population of late-phase stroke survivors can achieve functional gains by engaging in multimodal interventions. Participants in the H-RT group demonstrated immediate and sustained (6-month follow-up) improvement in short distance walking capacity, at both self-selected and fast speed. They walked the 10-meter distance faster and with fewer steps. In the R-MT group, an improvement was observed only at the 6-month follow-up, when they performed better at self-selected speed.

Walking speed was tested by timing a 10 -meter walk at self-selected but also fast speed, since that may be important in community-based activities such as crossing a street (Kollen et al., 2006). The immediate improvement in the timed 10-meter walk at self-selected gait speed for the H-RT group was 0.08 $( \pm 0.12) \mathrm{m} / \mathrm{s}$, and thus reached the level of $0.06 \mathrm{~m} / \mathrm{s}$, established as a small but meaningful change (Perera et al., 2006). At 6 months post-intervention, the gait speed gains for both the H-RT and R-MT group $(0.09 \mathrm{~m} / \mathrm{s})$ also represent a small but meaning- 
ful change. These results are comparable with the increase in gait speed (comfortable speed in 7 out of 9 included studies) by $0.07 \mathrm{~m} / \mathrm{s}$, described in a systematic review of gait training after stroke (Pappas \& Salem, 2009). Notably, cardiovascular improvement occurring in the later phase after stroke is conceivably limited due to the high energy demands of hemiplegic gait and the poor physical condition of stroke survivors (Ivey et al., 2006; Zamparo et al., 1995). The H-RT but not R-MT led to improvements in functional motor performance as measured by M-MAS UAS. The clinical relevance of this result is not clear, as the minimum detectable change and the minimal clinically important difference of the M-MAS UAS are yet to be determined (English et al., 2006).

H-RT and R-MT are specifically adapted to neurologically disabled individuals, including stroke survivors (Bunketorp-Käll et al., 2017; Lee et al., 2014). In H-RT, the three-dimensional movements of the horse stimulate several different muscle groups (Ribeiro et al., 2018), as well as the motor system (Garner \& Rigby, 2015). H-RT stimulates a similar rhythmical and repetitive pelvic movement of the rider as normal gait, which can provide important motor and sensory stimulus in persons with reduced mobility (Ribeiro et al., 2018). R-MT on the other hand, is based on exercises that challenge cognitive function and sensorimotor control, with the aim to improve coordination, mobility and cognition in patients with neurological deficits through the use of rhythm and music (Pohl et al., 2018a). The explicit motor component of H-RT may have resulted in the immediate and more pronounced effects of this intervention with regard to gait and functional mobility (Stergiou et al., 2017). ANCOVA theoretically yields unbiased estimates of intervention effect (Egbewale et al., 2014). However, in light of the trend towards between-group differences in baseline functional impairment, we cannot exclude that the smaller effect of R-MT observed in our study was at least partially due to a lower degree of functional impairment in the R-MT group.

We previously reported that the proportion of participants who felt they had achieved a meaningful recovery post-intervention was higher in the H-RT (56\%) compared with the R-MT group (38\%) (Bunketorp-Käll et al., 2017). These primary outcome findings are well in line with the results of the present study. At 6 months, the proportion of participants who felt they had achieved a meaningful recovery was 56 and $43 \%$, for the H-RT and R-MT group, respectively indicating a continued improvement among R-MT participants (BunketorpKäll et al., 2017). The post-intervention assessment further showed improvements in static/dynamic and functional balance ability, as measured by Berg Balance Scale (BBS) and Timed Up and Go (TUG) test, respectively in the H-RT group (Bunketorp-Käll et al., 2017). In the R-MT group, improvement was detected by Bäckstrand Dahlberg and Liljenäs (BDL) balance scale but not BBS (Bunketorp-Käll et al., 2017). The BDL scale is more demanding with regard to balance complexity, and is therefore more sensitive in detecting small improvement compared to BBS (Rosen et al., 2005).

The findings from both studies indicate that the motor component is more pronounced in HR-T, than R-MT. The H-RT may more specifically target the common coordination deficits after stroke, including altered temporal and spatial coordination between the head, trunk and pelvis(Hollands et al., 2012), that were suggested to affect gait execution (Daly et al., 2007). Moreover, H-RT is shown to reduce spasticity (Rigby \& Grandjean, 2016), which may facilitate various gait parameters (Hsu et al., 2003). The HRT participants in the present study were able to walk faster with fewer steps after the intervention, with a significant increase in step length at 6 months. This, may have been achieved by improved balance or lower limb coordination reported previously (Bunketorp-Käll et al., 2017).

The long-term positive effects of R-MT on balance, gait and working memory, as found in this and our previous study ${ }^{9}$ are also of importance as the improvement in these variables at the 6-month follow-up may reflect continued recovery after end of intervention. As interventions targeting lower limb coordination appear to improve walking for individuals with stroke (Hollands et al., 2012), the high demands of R-MT on coordination may have contributed to the improvement in gait capacity through the same mechanism.

In view of participants' own experiences of these multimodal interventions, both encounter enriching experiences that may have positive impacts on their emotional and physical state (Pohl et al., 2018a; Pohl et al., 2018b). The in-depth interviews indicate that the social interplay and the practical activities were considered as equally important among the participants themselves (Pohl et al., 2018b). As previously mentioned, certain limitations of our study must be considered when interpreting the results (BunketorpKäll et al., 2017). Due to the eligibility criteria in the RCT, our study population was limited to individuals 
with moderate impairment after stroke ( $\mathrm{mRS}$ grade 2 and 3). The study did not enrol individuals dependent on external help (mRS grade 4), as their possibility to actively participate in the interventions is limited and they often have other needs for support and rehabilitation. Whereas these individuals could potentially benefit from multimodal interventions like those used in our study, the, inclusion of individuals with minor residual disabilities would conceivably not be justifiable in terms of cost-effectiveness. Future research should aim to extend our findings to these groups and determine the cost-effectiveness of multimodal poststroke interventions. The present study has strengths such as the concealed design, stratified randomization, valid and reliable outcome measure, both an ITT and PP analysis and a low drop-out rate (BunketorpKäll et al., 2017). Given that improvements occurring in the later phase after stroke are considered to be barely noticeable (Duncan \& Min Lai, 1997), it is not surprising that the present study did not find any large effects of the interventions. Our findings, however, provide further support to the notion that potential for further recovery in functional mobility exists in late phase after stroke.

In conclusion, our results show that H-RT could be effective in producing improvement in gait and functional mobility in late-phase stroke survivors, whereas the effectiveness of R-MT is less clear. Further research needs to determine whether the benefits of H-RT are cost-effective and whether these conclusions can be extended to other populations of stroke survivors.

\section{Clinical trial registration}

ClinicalTrials.gov, NCT01372059.

\section{Acknowledgements}

The authors would like to acknowledge the physiotherapist, occupational therapist and assistants who carried out the H-RT and the certified therapist of the R-MT. We would also like to acknowledge the physiotherapists responsible for the scientific evaluation. Finally, we would like to acknowledge Aldina Pivodic for statistical guidance.

\section{Sources of funding}

This work was supported by grants from Sten A Olsson foundation for Research and Culture, the
Swedish Brain Foundation, the Swedish Arts Council, LUA/ALF Gothenburg, AFA Insurance, The Swedish Stroke Association, Rune and Ulla Amlöv's Foundation for Neurological and Rheumatological Research, Edith Jacobson Foundation, Per-OlofAhl Foundation for Neurological Research, Sigurd and Elsa Goljes Memorial Foundation, Wilhelm and Martina Lundgren Scientific Foundation, Doktor Felix Neubergh's Foundation, the Swedish Society of Medicine, the Foundation for Rehabilitation and Medical Science and the Gladys M. Brawn Fund at University of Newcastle.

\section{Conflict of interest}

The authors declare no potential conflict of interest with respect to the research, authorship and/or publication of this article.

\section{Supplementary material}

The supplementary file is available in the electronic version of this article: http://dx.doi.org/10.3233/ NRE-192905.

\section{References}

Adamson, J., Beswick, A., \& Ebrahim, S. (2004). Is stroke the most common cause of disability? Journal of Stroke and Cerebrovascular Diseases, 13(4), 171-177.

Alzahrani, M., Dean, C., \& Ada, L. (2011). Relationship between walking performance and types of community-based activities in people with stroke: An observational study. Brazilian Journal of Physical Therapy, 15(1), 45-51.

Andersson, C., \& Clevnert, M. (1997). Reliabilitetsprövning av Modifierad Motor Assessment Scale enligt Uppsala Akademiska sjukhus -99. Uppsala Universitet. HK-97. (Examensarbete 10p, C-nivå).

Barkelius, K., Johansson, A., Körm, K., \& Lindmark, B. (1997). Reliabilitets- och validitetsprövning av modifierad Motor Assessment Scale enligt Uppsala Akademiska sjukhus -95. Nordisk Fysioterapi (in Swedish), 1, 121-126.

Bernhardt, J., Borschmann, K., Boyd, L., Carmichael, S. T., Corbett, D., Cramer, S. C., Hoffmann, T., Kwakkel, G., Savitz, S., \& Saposnik, G. (2017a). Moving rehabilitation research forward: Developing consensus statements for rehabilitation and recovery research. Neurorehabilitation and Neural Repair, 31(8), 694-698.

Bernhardt, J., Borschmann, K., Boyd, L., Carmichael, S. T., Corbett, D., Cramer, S. C., Hoffmann, T., Kwakkel, G., Savitz, S., Saposnik, G., Walker, M., \& Ward, N. (2017b). Moving Rehabilitation Research Forward: Developing Consensus Statements for Rehabilitation and Recovery Research. Neurorehabil Neural Repair, 31(8), 694-698. 
Bernhardt, J., Hayward, K. S., Kwakkel, G., Ward, N. S., Wolf, S. L., Borschmann, K., Krakauer, J. W., Boyd, L. A., Carmichael, S. T., Corbett, D., \& Cramer, S. C. (2017c). Agreed definitions and a shared vision for new standards in stroke recovery research: The Stroke Recovery and Rehabilitation Roundtable taskforce. Int J Stroke, 12(5), 444-450.

Brandstater, M. E., Gowland, C., \& Clark, B. (1983). Hemiplegic gait: Analysis of temporal variables. Archives of Physical Medicine and Rehabilitation, 64(12), 583-587.

Bunketorp-Käll, L., Lundgren-Nilsson, ̊., Samuelsson, H., Pekny, T., Blomvé, K., Pekna, M., Pekny, M., Blomstrand, C., \& Nilsson, M. (2017). Long-term improvements after multimodal rehabilitation in late phase after stroke: A randomized controlled trial. Stroke, 48(7), 1916-1924.

Bunketorp Kall, L., Lundgren-Nilsson, A., Blomstrand, C., Pekna, M., Pekny, M., \& Nilsson, M. (2012). The effects of a rhythm and music-based therapy program and therapeutic riding in late recovery phase following stroke: A study protocol for a three-armed randomized controlled trial. BMC Neurology, 12(1), 141

Carr, J. H., Shepherd, R. B., Nordholm, L., \& Lynne, D. (1985). Investigation of a new motor assessment scale for stroke patients. Physical Therapy, 65(2), 175-180.

Choi, M., Yoo, J., Shin, S., \& Lee, W. (2015). The effects of stepper exercise with visual feedback on strength, walking, and stair climbing in individuals following stroke. Journal of Physical Therapy Science, 27(6), 1861-1864.

Collen, F. M., Wade, D. T., \& Bradshaw, C. M. (1990). Mobility after stroke: Reliability of measures of impairment and disability. International Disability Studies, 12(1), 6-9.

Cooper, K. H. (1968). A means of assessing maximal oxygen intake: Correlation between field and treadmill testing. JAMA, 203(3), 201-204.

Corbett, D., Jeffers, M., Nguemeni, C., Gomez-Smith, M., \& Livingston-Thomas, J. (2015). Lost in translation: Rethinking approaches to stroke recovery. Prog Brain Res, 218, 413-434.

Corbyn, Z. (2014). Stroke: A growing global burden. Nature, 510(7506), S2-S3.

Daly, J. J., \& Ruff, R. L. (2007). Construction of efficacious gait and upper limb functional interventions based on brain plasticity evidence and model-based measures for stroke patients. Scientific World Journal, 7, 2031-2045.

Daly, J. J., Sng, K., Roenigk, K., Fredrickson, E., \& Dohring, M. (2007). Intra-limb coordination deficit in stroke survivors and response to treatment. Gait and Posture, 25(3), 412418.

Demain, S., Wiles, R., Roberts, L., \& McPherson, K. (2006). Recovery plateau following stroke: Fact or fiction? Disability and Rehabilitation, 28(13-14), 815-821.

Dickstein, R. (2008). Rehabilitation of gait speed after stroke: A critical review of intervention approaches. Neurorehabilitation and Neural Repair, 22(6), 649-660.

Duncan, P. W., \& Min Lai, S. (1997). Stroke recovery. Topics in stroke Rehabilitation, 4(3), 51-58.

Duncan, P. W., Sullivan, K. J., Behrman, A. L., Azen, S. P., Wu, S. S., Nadeau, S. E., Dobkin, B. H., Rose, D. K., Tilson, J. K., \& Team, L. I. (2007). Protocol for the Locomotor Experience Applied Post-stroke (LEAPS) trial: A randomized controlled trial. BMC Neurology, 7, 39.

e Silva, E. M. G. d. S., Ribeiro, T. S., da Silva, T. C. C., Costa, M. F. P., Cavalcanti, F. A. d. C., \& Lindquist, A. R. R. (2017). Effects of constraint-induced movement therapy for lower limbs on measurements of functional mobility and postural balance in subjects with stroke: A randomized controlled trial. Topics in stroke Rehabilitation, 24(8), 555-561.

Egbewale, B. E., Lewis, M., \& Sim, J. (2014). Bias, precision and statistical power of analysis of covariance in the analysis of randomized trials with baseline imbalance: A simulation study. BMC Medical Research Methodology, 14(1), 49.

English, C. K., Hillier, S. L., Stiller, K., \& Warden-Flood, A. (2006). The sensitivity of three commonly used outcome measures to detect change amongst patients receiving inpatient rehabilitation following stroke. Clinical Rehabilitation, 20(1), 52-55.

Garner, B. A., \& Rigby, B. R. (2015). Human pelvis motions when walking and when riding a therapeutic horse. Human movement science, 39, 121-137.

Harris, J. E., \& Eng, J. J. (2004). Goal Priorities Identified through Client-Centred Measurement in Individuals with Chronic Stroke. Physiotherapy Canada, 56(3), 171-176.

Hesse, S., Werner, C., Paul, T., Bardeleben, A., \& Chaler, J. (2001). Influence of walking speed on lower limb muscle activity and energy consumption during treadmill walking of hemiparetic patients. Archives of Physical Medicine and Rehabilitation, 82(11), 1547-1550.

Hollands, K. L., Pelton, T. A., Tyson, S. F., Hollands, M. A., \& van Vliet, P. M. (2012). Interventions for coordination of walking following stroke: Systematic review. Gait and Posture, 35(3), 349-359.

Hsu, A.-L., Tang, P.-F., \& Jan, M.-H. (2003). Analysis of impairments influencing gait velocity and asymmetry of hemiplegic patients after mild to moderate stroke1. Archives of Physical Medicine and Rehabilitation, 84(8), 1185-1193.

Ivey, F. M., Hafer-Macko, C. E., \& Macko, R. F. (2006). Exercise rehabilitation after stroke. NeuroRx, 3(4), 439-450.

Jette, D. U., Latham, N. K., Smout, R. J., Gassaway, J., Slavin, M. D., \& Horn, S. D. (2005). Physical therapy interventions for patients with stroke in inpatient rehabilitation facilities. Physical Therapy, 85(3), 238-248.

Johansson, B. B. (2012). Multisensory stimulation in stroke rehabilitation. Frontiers in Human Neuroscience, 6, 60. doi: 10.3389/fnhum.2012.00060. eCollection 02012.

Kollen, B., Kwakkel, G., \& Lindeman, E. (2006). Hemiplegic gait after stroke: Is measurement of maximum speed required? Archives of Physical Medicine and Rehabilitation, 87(3), 358363.

Lee, C. W., Kim, S. G., \& Yong, M. S. (2014). Effects of hippotherapy on recovery of gait and balance ability in patients with stroke. J Phys Ther Sci, 26(2), 309-311.

Mala, H., \& Rasmussen, C. P. (2017). The effect of combined therapies on recovery after acquired brain injury: Systematic review of preclinical studies combining enriched environment, exercise, or task-specific training with other therapies. Restor Neurol Neurosci, 35(1), 25-64.

Michael, K. (2002). Fatigue and stroke. Rehabilitation Nursing, 27(3), 89-94, 103.

Nagano, K., Hori, H., \& Muramatsu, K. (2015). A comparison of at-home walking and 10-meter walking test parameters of individuals with post-stroke hemiparesis. Journal of Physical Therapy Science, 27(2), 357-359.

Pappas, E., \& Salem, Y. (2009). Overground physical therapy gait training for chronic stroke patients with mobility deficits. Cochrane Database Syst Rev (3). 
Perera, S., Mody, S. H., Woodman, R. C., \& Studenski, S. A. (2006). Meaningful change and responsiveness in common physical performance measures in older adults. Journal of the American Geriatrics Society, 54(5), 743-749.

Perry, J., Garrett, M., Gronley, J. K., \& Mulroy, S. J. (1995). Classification of walking handicap in the stroke population. Stroke, 26(6), 982-989.

Pohl, P., Carlsson, G., Käll, L. B., Nilsson, M., \& Blomstrand, C. (2018a). Experiences from a multimodal rhythm and musicbased rehabilitation program in late phase of stroke recovery-A qualitative study. PloS One, 13(9), e0204215.

Pohl, P., Carlsson, G., Käll, L. B., Nilsson, M., \& Blomstrand, C. (2018b). A qualitative exploration of post-acute stroke participants' experiences of a multimodal intervention incorporating horseback riding. PloS One, 13(9), e0203933.

Poole, J. L., \& Whitney, S. L. (1988). Motor assessment scale for stroke patients: Concurrent validity and interrater reliability. Archives of Physical Medicine and Rehabilitation, 69(3 Pt 1), 195-197.

Rand, D., Eng, J. J., Tang, P.-F., Jeng, J.-S., \& Hung, C. (2009). How active are people with stroke? Use of accelerometers to assess physical activity. Stroke, 40(1), 163-168.

Ribeiro, M. F., Espindula, A. P., Júnior, D. E. B., Tolentino, J. A., da Silva, C. F. R., Araújo, M. F., Ferreira, A. A., \& Teixeira, V. d. P. A. (2018). Activation of lower limb muscles with different types of mount in hippotherapy. Journal of Bodywork and Movement Therapies, 22(1), 52-56.

Rigby, B. R., \& Grandjean, P. W. (2016). The Efficacy of Equine-Assisted Activities and Therapies on Improving Physical Function. The Journal of Alternative and Complementary Medicine, 22(1), 9-24.

Rosen, E., Sunnerhagen, K. S., \& Kreuter, M. (2005). Fear of falling, balance, and gait velocity in patients with stroke. Physiotherapy Theory and Practice, 21(2), 113-120.

Schulz, K. F., Altman, D. G., Moher, D., \& Group, C. (2010). CONSORT 2010 Statement: Updated guidelines for reporting parallel group randomised trials. BMC Medicine, 8, 18.
Stergiou, A., Tzoufi, M., Ntzani, E., Varvarousis, D., Beris, A., \& Ploumis, A. (2017). Therapeutic Effects of Horseback Riding Interventions: A Systematic Review and Meta-analysis. American Journal of Physical Medicine and Rehabilitation, 96(10), 717-725.

Swisher, A. K., \& Goldfarb, A. H. (1998). Use of the Six-Minute Walk/Run Test to Predict Peak Oxygen Consumption in Older Adults. Cardiopulmonary Physical Therapy Journal, 9(3), 3-5.

Tyson, S., \& Connell, L. (2009). The psychometric properties and clinical utility of measures of walking and mobility in neurological conditions: A systematic review. Clinical Rehabilitation, 23(11), 1018-1033.

Tyson, S. F., \& Rogerson, L. (2009). Assistive walking devices in nonambulant patients undergoing rehabilitation after stroke: The effects on functional mobility, walking impairments, and patients' opinion. Archives of Physical Medicine and Rehabilitation, 90(3), 475-479.

Wall, J. C., \& Turnbull, G. I. (1986). Gait asymmetries in residual hemiplegia. Archives of Physical Medicine and Rehabilitation, 67(8), 550-553.

van de Port, I. G., Kwakkel, G., \& Lindeman, E. (2008). Community ambulation in patients with chronic stroke: How is it related to gait speed? Journal of Rehabilitation Medicine, 40(1), 23-27.

Veerbeek, J. M., van Wegen, E., van Peppen, R., van der Wees, P. J., Hendriks, E., Rietberg, M., \& Kwakkel, G. (2014). What is the evidence for physical therapy poststroke? A systematic review and meta-analysis. PloS One, 9(2), e87987.

Zamparo, P., Francescato, M., De Luca, G., Lovati, L., \& di Prampera, P. (1995). The energy cost of level walking in patients with hemiplegia. Scandinavian Journal of Medicine and Science in Sports, 5(6), 348-352.

Öberg, T., Karsznia, A., \& Öberg, K. (1993). Basic gait parameters: Reference data for normal subjects, 10-79 years of age. Journal of Rehabilitation Research and Development, 30, 210-210. 